\title{
Superparamagnetism and Monte Carlo Simulations
}

\author{
D. Serantes and D. Baldomir*
}

\begin{abstract}
Departamento de Física Aplicada and Instituto de Investigacións Tecnolóxicas, Universidade de Santiago de Compostela, Santiago de Compostela E-15782, Galiza, Spain
\end{abstract}

\begin{abstract}
We summarize the basic concepts beneath the idea of superparamagnetism and introduce the Monte Carlo (MC) method as a powerful tool for studying superparamagnetic (SPM) properties. Starting with the description of the physical features of the single-domain SPM entities, we concentrate on their special magnetic properties as a function of time, temperature, and applied magnetic field. Then, we give a general approach to the use of a MC technique for studying SPM properties, focusing on the use of the Metropolis algorithm and discussing the importance of the choice of the MC steps in the simulations.
\end{abstract}

Keywords: Magnetic nanoparticles, superparamagnetism, monte carlo simulations, magnetic anisotropy, blocking temperature, single-domain particles.

\section{INTRODUCTION}

Nanosized magnetic materials exhibit a rich variety of magnetic phenomena in comparison with the bulk counterparts, what gives place to a novel range of applications of great importance to improve daily human activities as high-density magnetic recording storage or biomedical applications [1]. The origin of these special magnetic properties is found on the reduced dimensionality [2]: when the size of the material reaches the order of nanometers the influence of the surface atoms becomes comparable (or even more important) than the bulk contribution; the defects associated to the broken crystalline symmetry may be of significant importance, and other physical effects may also become very relevant when the size reaches the order of characteristic length scales of the material. The physical properties observed at such reduced dimensions are strongly sensitive to slight variations of size, shape, and composition. Therefore, different magnetic structures (nanoparticles, nanoparticle arrays, nanowires, thin films, etc.) constitute differentiated research fields with singular characteristics [3].

In the first part of this article we revise the concept of superparamagnetism, one of the most remarkable magnetic properties that arises in these reduced dimensions. Superparamagnetic (SPM) phenomenon stands for the paramagnetic-like behavior displayed by single-domain magnetic entities above a characteristic threshold named blocking temperature, which is determined by a complex interplay between the intrinsic physical characteristics of the material (magnetic moment, anisotropy, etc.) and the experimental conditions (measuring time, applied magnetic field, etc.). Understanding the SPM properties of nanosized systems is of primordial importance both for the basic

*Address correspondence to this author at the Departamento de Física Aplicada and Instituto de Investigacións Tecnolóxicas, Universidade de Santiago de Compostela, Santiago de Compostela E-15782, Galiza, Spain; Tel: 0034881813969; Fax: 881814112; E-mail: daniel.baldomir@usc.es theoretical knowledge [4] and for specific-designed applications (as for example the increase of the storage information capacity of hard drives [5], or the development of well-controlled biomedical applications [6]). Due to the above reasons, a big effort has been devoted to the study of SPM systems in the last years, aimed to understand its underlying physical mechanisms.

However, the investigation of SPM properties is a complex task due to its strong dependence on several uncontrolled parameters, which mask the physical origin of the magnetic behavior and hence make very difficult to achieve a precise characterization. The large dispersion in the value of the parameters (particle size, anisotropy, shape) found in real systems, and the presence of uncontrolled interparticle interactions, results in a complex physical problem non-solvable by analytical methods. To investigate such scenario it is very common the use of computational techniques, which allow a precise control of the physical parameters governing the system: by means of a computational technique it is possible to set up ideal systems (e.g. monodisperse size and/or anisotropy) specially designed to ease the comprehension of a particular physical mechanism. In the second part of this work we introduce the basic characteristics of a Monte Carlo (MC) method based on the Metropolis algorithm to undertake the study of SPM properties.

\section{SUPERPARAMAGNETISM}

The term superparamagnetism refers to the magnetic phenomena observed in fine magnetic particle systems exhibiting close similarities to atomic paramagnetism. Basically, single-domain magnetic nanoparticles can be characterized by their large total magnetic supermoment, which exhibits a paramagnetic-like behavior above a characteristic temperature threshold named blocking temperature, $T_{B}$. This particular temperature, in contraposition with the Curie temperature $T_{C}$, is extremely dependent on the experimental observational time-window and this characteristic gives place to a complex theoretical 
frame with especial experimental features. In what follows we briefly introduce the conditions for the existence of superparamagnetism and its basic characteristics. For more information in this topic see for example the reviews by D. Kechrakos [7], O. Petracic [8], M. Knobel et al. [9], J.L. Dormann et al. [4].

\subsection{Single-Domain Particles}

SPM phenomenon is observed upon reduction of the size of ferromagnetic (FM) materials ${ }^{1}$ down to the single-domain range. In a FM magnetic material, multiple magnetic domains exist as a result of the balance between the exchange interaction energy that favors the parallel alignment of neighboring atomic moments (thereby forming magnetic domains), and the magnetostatic interaction energy that tries to break them into smaller domains oriented antiparallel to each other. The domain size is determined by the relative counterbalance between both energies. With decreasing size of the magnetic system, there is a critical value ( $r_{c}$ for the radius of a spherical particle) below which the magnetostatic energy no longer allows for the breaking of the system into smaller domains and so the system is composed of a single domain, as illustrated in Fig. (1). Typical values for $r_{c}$ are of the order of tens of nanometers.
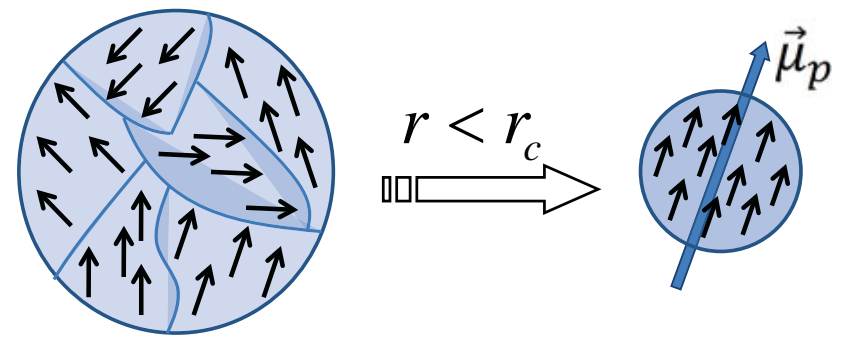

Fig. (1). Scheme illustrating the transition from the multi-domain configuration to the single-domain one upon size reduction.

Assuming coherent rotation of the atomic magnetic moments within the single-domain structure, the particle can be characterized by its total magnetic supermoment, $\vec{\mu}_{p}$. In first approximation, considering uniform magnetization (neglecting surface effects) it can be described as proportional to the particle volume $V$ and saturation magnetization $M_{S}$ as

$\left|\vec{\mu}_{p}\right|=M_{S} V$

As mentioned above, the SPM response in magnetic nanoparticles is observed above the blocking temperature, $T_{B}$, a proper feature of SPM systems that differentiates them from atomic paramagnetism. The origin of $T_{B}$ relays on the magnetic anisotropy present within the nanoparticles (unlike isolated atomic moments), which tends to orientate the particle supermoment along some preferential direction. The

\footnotetext{
1 The term "ferromagnetic" refers here to any magnetic configuration resulting in a net total magnetic moment, regardless the atomic arrangement, to be ferro-, ferri-, canted, etc. The important characteristic is the existence of a non-zero magnetic moment configuration in absence of applied magnetic field.
}

magnetic anisotropy appears due to the finite size of the particles, which are made up of several atoms (usually up to thousands of atoms for single-domain particles), and thus spin-orbit coupling and dipolar interaction dictate preferential orientation directions for the magnetic moments. Depending on the specific characteristics of the particles (electronic structure, shape) the role played by each anisotropy source will change and give rise to complex anisotropy pictures. Often, the magnetic anisotropy energy $E_{A}$ of the particles can be described by a simple model with two main contributions, crystalline and shape, which are associated to the core and surface atoms, respectively (for details about different anisotropy sources see for example ref. [4]). For the sake of simplicity, we have focused on the simplest uniaxial anisotropy case, very reasonable assumption for a spherical particle with uniaxial crystalline anisotropy [11]. From now on we consider the different anisotropy contributions as comprised in an effective uniaxial anisotropy term, $\vec{K}_{\text {eff }}$, as illustrated in Fig. (2a).

If we consider the magnetic anisotropy to be proportional to the particle volume as $\vec{K}_{\text {eff }}=K V \hat{n}$, with $K$ the effective uniaxial anisotropy constant (per unit volume) and $\hat{n}$ the unitary vector describing the easy-magnetization anisotropy direction, then the energy term for the $i$-particle can be written as

$$
E_{A}^{(i)}=-K_{i} V_{i}\left(\frac{\vec{\mu}_{i} \cdot \hat{n}_{i}}{\left|\vec{\mu}_{i}\right|}\right)^{2}=-K_{i} V_{i} \cos ^{2} \theta_{i}
$$

where $\theta_{i}$ is the angle between the magnetic supermoment of the particle and the easy anisotropy axis. The moment of the particle has therefore two preferred orientations, energetically equivalent, along the easy-magnetization anisotropy axis direction. Both directions are separated by an energy barrier $E_{B}$ of height $K_{i} V_{i}$. The energy spectra corresponding to this uniaxial anisotropy energy is illustrated in Fig. (2b).

The system we have constructed up to now is that of homogeneous magnetic nanoparticles characterized by their size $V$, saturation magnetization $M_{S}$, and uniaxial magnetic anisotropy energy $K$. This very simple scenario describes reasonably well many experimental situations, and therefore from now on we focus on the analysis of the magnetic properties of such particle system as a function of temperature $(T)$ and magnetic field $(\vec{H})$. Real systems are usually characterized by randomness in their spatial distribution and in the easy-axes orientation that strongly influence the properties of the system as determined by interparticle-interactions and applied magnetic field, what results in a complex problem. For the sake of simplicity we consider for the moment a non-interacting system with parallel aligned anisotropy easy axes, so that the particles are equivalent to each other and the system can be studied under a single-particle perspective. 
(a)
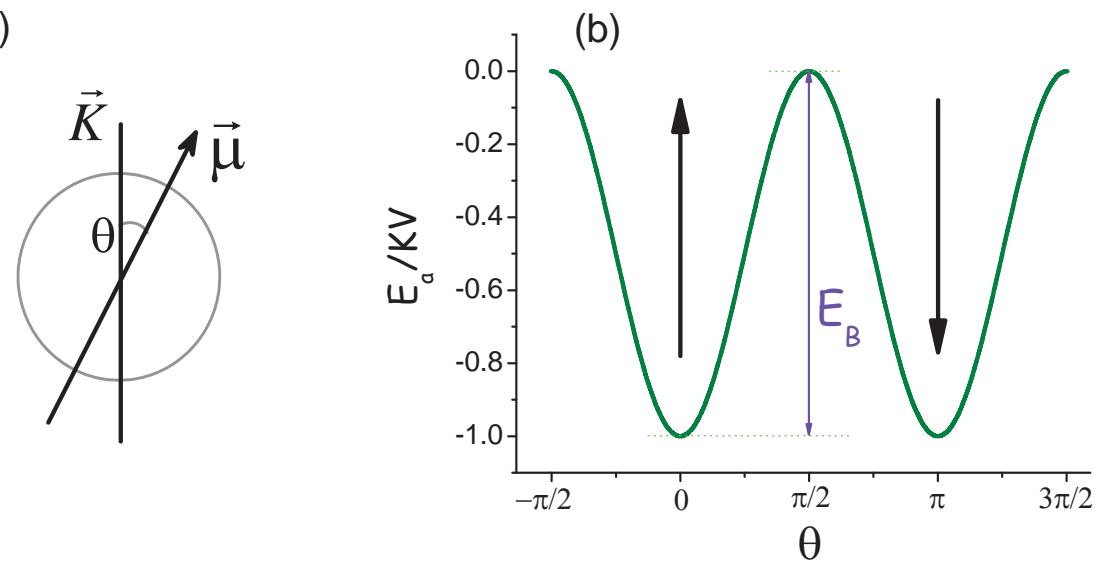

Fig. (2). (a) Schematic drawing of the uniaxial magnetic anisotropy $K$ and magnetic supermoment of a single-domain nanoparticle, and (b) the corresponding uniaxial anisotropy wells.

\subsection{Thermal Relaxation and Blocking Temperature $\left(T_{B}\right)$}

The thermal energy promotes the fluctuation of the magnetic moments, and therefore to understand the role that the temperature plays on the magnetic behavior of the particles it is necessary to investigate the dynamics of the particle moments as a function of T. The high- and low- T limit cases can be easily figured out: at very high $\mathrm{T}$, the thermal energy is much larger than the anisotropy energy barrier ( $k_{B} T>>E_{B}$, with $k_{B}$ the Boltzmann constant) and so the magnetic anisotropy plays a negligible influence on the orientation of the magnetic moments of the particles, which fluctuate freely with temperature. In this case, a paramagnetic-like behavior is observed and the particles are in the superparamagnetic state (SPM state). On the contrary, at very low $\mathrm{T}$ the particle moment remains confined along the anisotropy direction (local energy minimum) because thermal fluctuations are unable to switch its orientation out of that minimum $\left(k_{B} T<<E_{B}\right)$. When this happens the particles are said to be in the blocked state (BL state).

It is much more complicated to describe the influence of thermal fluctuation on the orientation of the particles' supermoments for the intermediate- $\mathrm{T}$ cases. This was first described by Néel [10], who proposed that the thermal fluctuations could promote the jumping of the magnetic moment of the particles from one anisotropy well to the other, introducing the average time $\tau$ for thermal activation (often called relaxation time) over the anisotropy barrier to follow an Arrhenius law

$$
\tau=\tau_{0} e^{\frac{E_{B}}{k_{B} T}}
$$

where $\tau_{0}$ directly depends on the material parameters ( $K$, $M_{S}$, etc) and is of the order of $10^{-11}-10^{-9} \mathrm{~s}$. Under this description, it points out that the measuring time $\tau_{m}$ will be a key-point on determining the magnetic state of the system: if the measuring time is large in comparison with the characteristic reversal time of the particles, $\tau_{m} \gg \tau$, then the particle moment will fluctuate freely from one well to the other in a paramagnetic-like manner, i.e. the particle will be in the SPM state. However, if $\tau_{m}<<\tau$, during the measuring time the particle moment will be unable to jump over the anisotropy barrier and hence will remain blocked along one anisotropy well, i.e. the particle will be in the BL state. Macroscopically, the SPM state is completely reversible upon temperature and field variations, whereas the $\mathrm{BL}$ one is characterized by its ferromagnetic-like hysteretic behavior. The limit between both states is found at $\tau_{m} \equiv \tau$, and serves for the definition of $T_{B}$, as illustrated in Fig. (3) and obtained from Eq. (3)

$$
T_{B}=\frac{K V}{k_{B} \ln \left(\tau_{m} / \tau_{0}\right)}
$$

Eq. (4) shows how $T_{B}$ depends both on the intrinsic particle parameters and on the measuring time. By varying the external influences of the systems (temperature, measuring time, magnetic field) we may tune its response and can extract information about the characteristics of the system from the trends obtained.

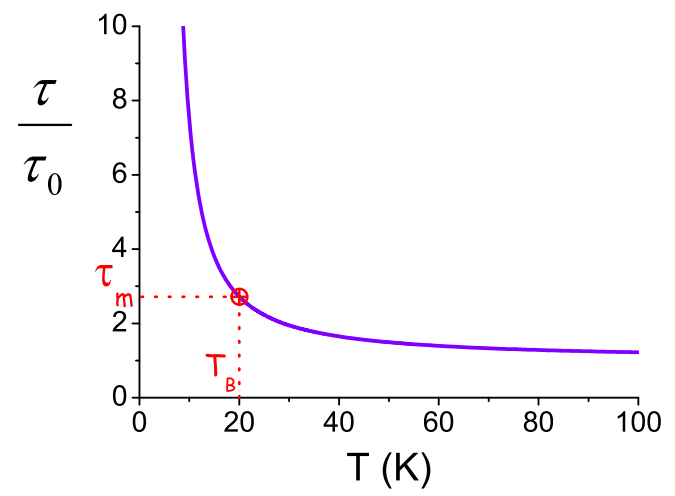

Fig. (3). Relaxation time $\tau$ vs $\mathrm{T}$, and indication of $T_{B}$ for a certain $\tau_{m}$.

Eq. (4) highlights that the value of $\tau_{m}$ is decisive in determining $T_{B}$ and hence the magnetic response of the system in a given time-scale (for example, for informationstorage purposes very large time scales are necessary, contrary to the very short times required for magnetic recording). Therefore, the choice of the experimental 
technique will vary depending on the particular objective, since it determines the value of $\tau_{m}$. In this work we have focused on quasistatic processes, and so associate $T_{B}$ with the one obtained in dc-thermomagnetizatic measurements, in which the measuring time is very large $\left(\tau_{m} \approx 100 s\right)$ in comparison with the characteristic time $\tau_{0}$ of the particles. Other measurement techniques involving much shorter measuring times are associated to dynamic measurements, not considered in this work (for a recent review on the dynamic properties of SPM systems see for example ref. [11]; other measurement techniques associated to different time scales are analyzed in ref. [4]). We have mainly focused on data obtained following the standard zero field cooling (ZFC) and field cooling (FC) protocols, in which the system is perturbed under a low magnetic field for measuring the evolution of its magnetization with temperature. Before that, we analyze the effect of the magnetic field on the magnetic properties of the single-domain nanoparticles.

\subsection{Field Dependence}

When an external magnetic field is applied over the nanoparticles, it tries to align their magnetic moments along its direction. Therefore, except if applied perpendicularly to the easy anisotropy axis, it will favor the occupancy of one of the anisotropy energy wells over the other. The orientation of the magnetic moment of a particle $i$ is then governed by the competition among its anisotropy (uniaxial in the present case) and Zeeman energies, $E_{A}$ and $E_{Z}$, respectively:

$E^{i}=E_{A}^{(i)}+E_{Z}^{(i)}=-K_{i} V_{i}\left(\frac{\vec{\mu}_{i} \cdot \hat{n}_{i}}{\left|\vec{\mu}_{i}\right|}\right)^{2}-\vec{\mu}_{i} \cdot \vec{H}$

The influence of the external field in the orientation of the magnetic supermoments is known as the Stoner-Wohlfart model [12] after the authors who first considered and solved this problem. They assumed coherent rotation of the atomic magnetic moments and that the field was applied at a certain angle $\theta_{0}$ with respect to the easy anisotropy axis. By ignoring thermal effects they could solve the problem from minimal energy arguments, not taking into account timedependence as related to temperature. The aim of this introduction section is to give an overall description of the SPM features regarding the evolution of the magnetic moments with temperature in relation to the anisotropy energy barrier and $T_{B}$. Therefore, in the current section we do not discuss now the orientation of the field at different angles and focus, for the sake of simplicity, on the simple case of the field applied parallel to the easy anisotropy axis. Note that in this context of non-interacting particles, applying the magnetic field at a certain angle with respect to the easy axis is equivalent to consider only its projection along the axis. Since we are under the assumption of noninteracting and equivalent particles, we can apply singleparticle considerations and simplify the $i$ subindex in Eq. (5), which taking into account Eq. (1), reads

$E=-K V \cos ^{2} \theta-M_{S} V H \cos \theta$
For fields $H<2 K / M_{S}$, Eq. (6) has two local energy minima (easy magnetization directions) at $\theta=0, \pi$ with values $E_{\text {min }}=-K V \pm M_{S} V H$, and a maximum (hard magnetization direction) at $\theta=\arccos \left(H M_{S} / 2 K\right)$, with value $E_{\max }=K V\left(H M_{S} / 2 K\right)^{2}$. For the case $H=0$, the hard direction is the perpendicular to the anisotropy axis. The $\theta=0$ value stands for the parallel orientation of the particle moment with respect to the magnetic field ( $\uparrow \uparrow$ ), whereas the $\theta=\pi$ value stands for the antiparallel one $(\uparrow \downarrow)$. This difference in the shape of the energy wells, illustrated in Fig. (2), corresponds to different energy barriers depending on the orientation of the particle moment with respect to the applied field, which we name as $E_{B}^{\uparrow \downarrow}$ and $E_{B}^{\uparrow \uparrow}$ for the antiparallel and parallel cases, respectively. Introducing the anisotropy field of the particles as

$H_{A}=\frac{2 K}{M_{S}}$

we calculate these energy barriers as the difference between the minima and maximum energies, obtaining

$E_{B}^{\uparrow \downarrow}=K V\left(1-\frac{H}{H_{A}}\right)^{2}$

and

$E_{B}^{\uparrow \uparrow}=K V\left(1+\frac{H}{H_{A}}\right)^{2}$

The difference in the height of the energy barriers indicates also a change in the characteristic relaxation time of the particles, since it depends now on the relative orientation of the magnetic dipoles with respect to the field: particles antiparallel-oriented with respect to the field have a smaller energy barrier in comparison to the solely-anisotropy one and so a smaller thermal energy is enough to overcome it, whereas the parallel-oriented particles are now confined into a deeper anisotropy well and so a higher thermal energy is necessary to promote the jumping of its magnetic moments. This influence of the magnetic field on the energy wells of Fig. (2) is shown in Fig. (4, left panel), as well as its implications regarding the relaxation time of the particles (right panel).

Fig. (4) illustrates the importance of the strength of the applied magnetic field on determining the magnetic properties of SPM systems, and also serves as a definition of small field as referred to magnetic nanoparticles in comparison with their anisotropy field: the ratio $H / H_{A}$ must be as small as possible so that the system keeps as close as possible to the ideal SPM conditions.

\subsection{Thermomagnetic Measurements}

Once we have gone through how a magnetic field $\vec{H}$ influences the properties of the particles, we can undertake the description of the $Z F C$ and $F C$ measurements. In both processes the temperature evolution of the total magnetization of the system is recorded following different 

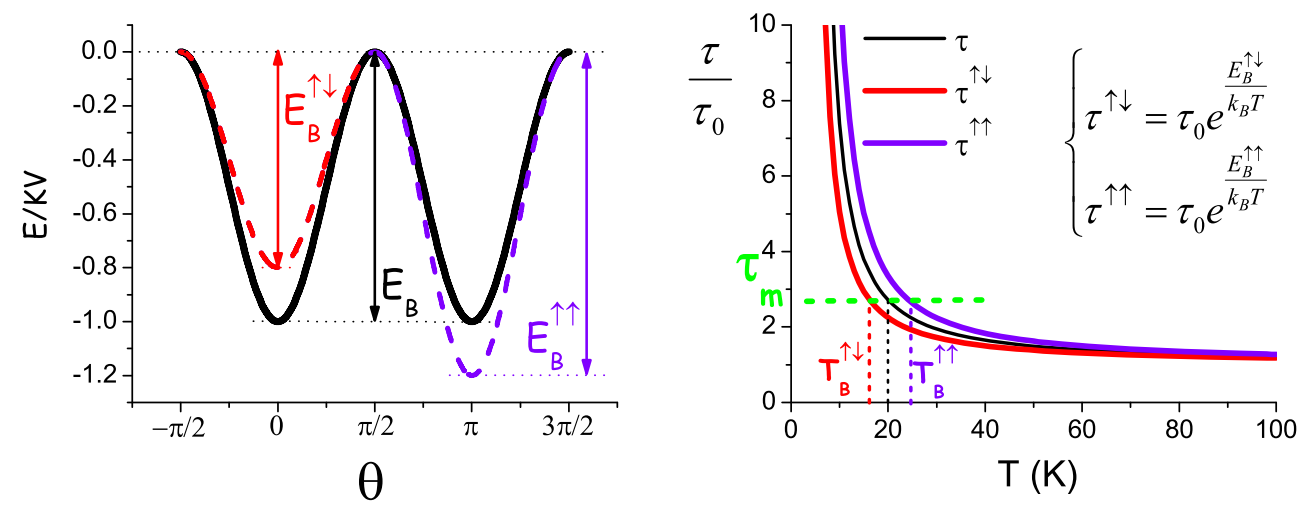

Fig. (4). Anisotropy energy wells (left panel) and relaxation time (right panel) of the particles as influenced by the magnetic field.

thermomagnetic histories, and it is this different history what highlights reversibility (no-hysteresis) and irreversibility (hysteresis) for differentiating the anhysteretic SPM state from the hysteretic BL state ${ }^{2}$. In a ZFC process, the system is first cooled down in zero field until a very low $\mathrm{T}$ is reached, and afterwards a small field is applied and the magnetization is recorded while heating the sample up. The FC curve is obtained by measuring the magnetization of the sample while cooling under low magnetic field (same field strength for both ZFC and FC processes). Typical ZFC/FC curves are shown in Fig. (5).

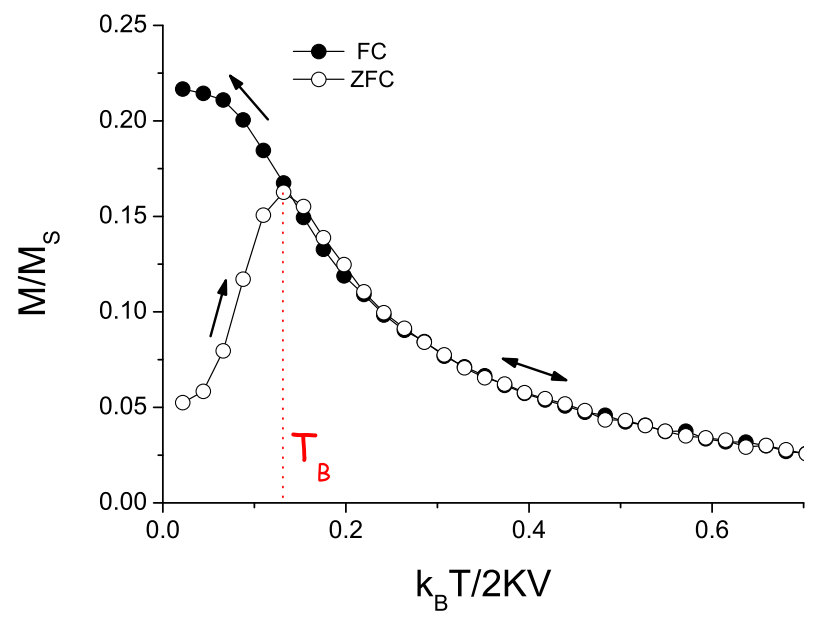

Fig. (5). Typical ZFC and FC magnetization curves vs temperature, presented in usual normalized units of $M / M_{S}$ and $k_{B} T / 2 K V$ (see

Subsection 3.4). Vertical dotted line stands for the maximum of the ZFC curve, usually associated to $T_{B}$.

The ZFC and FC curves shown in Fig. (5) display the usual features found in SPM systems: i) both curves coincide at high temperatures in a PM-like dependence; ii) with decreasing $\mathrm{T}$ both curves grow until a certain temperature range is reached where the curves start to diverge, the FC curve still growing although at a lower rate while the ZFC exhibits a maximum and decreases below it. This maximum

2 Although for evaluating magnetic hysteresis, to perform measurements of $\mathrm{M}(\mathrm{H})$ cycles could seem a better choice, they are not appropriate for determining the value of $T_{B}$, since it would be necessary to record $M(H)$ curves at several temperatures in order to obtain its value with enough precision. On the contrary, $\mathrm{M}(\mathrm{T})$ measurements give a continuous temperature range that allows to obtain $\mathrm{T}_{\mathrm{B}}$ easily, from a single curve. in the ZFC curve is generally associated in the literature to $T_{B}$ [7], as indicated in the figure, since such maximum roughly differentiates two main temperature regimes: a hightemperature one where both curves essentially coincide and exhibit PM-like temperature dependence, from a lowtemperature regime where both curves clearly diverge. However, a detailed view of the curves reveals that a $1 / T$ PM-like decrease right above $T_{B}$ is not observed in the ZFC curve, and a slight difference between the $\mathrm{ZFC}$ and $\mathrm{FC}$ one is perceived. These features indicate that a true SPM behavior is not exhibited right above the maximum, but at higher temperatures at which the ZFC curve perfectly overlaps the FC one and exhibits well defined PM-like temperature dependence. The reason why the ideal SPM behavior is not observed right at $T>T_{B}$ is found on the inverse of the relaxation time $\tau$, which gives the probability per unit time for the particle to overcome the anisotropy energy barrier along the temperature

$$
p(T)=\tau_{0}^{-\frac{-E_{B}}{k_{B} T}}
$$

Considering the different energy barriers $E_{B}^{\uparrow \downarrow}$ and $E_{B}^{\uparrow \uparrow}$ and so different relaxation times (see Fig. 4), Eq. (10) indicates that SPM behavior will be only observed (ideally) above $T_{B}^{\uparrow \uparrow}$, which is bigger than the maximum of the ZFC curve. Therefore, the features displayed in Fig. (4) concerning the different height of the anisotropy wells as influenced by the external field, must be taken into account too when analyzing the physical trend followed in the ZFC magnetization curve. The initial state of the ZFC process starts with no net magnetization after cooling in zero field. If naming parallel particles those with $E_{Z}<0$, and antiparallel particles those with $E_{Z}>0$, then when the field is applied the parallel particles will rapidly align with the field, while the antiparallel ones will progressively overcome the energy barrier with the increasing thermal energy and also align with the field. This process leads to a continuous increase of the magnetization, as illustrated in Fig. (5), until the thermal energy overcomes $E_{B}^{\uparrow \downarrow}$, and so no longer reversal magnetization takes place. Since the thermal energy is now comparable to the energy of the deeper anisotropy well, the particles start to fluctuate freely with increasing the 


\section{Parallel easy axes}
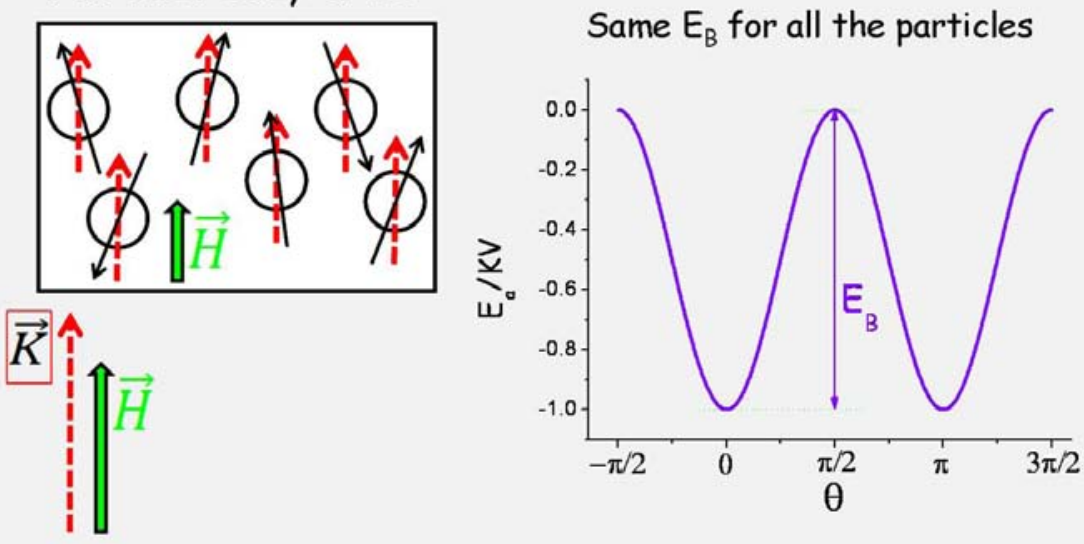

Fig. (6). Schematic drawing of the ideal simplest model of non-interacting and parallel aligned easy axes along the applied field.

temperature and thus a decrease in the magnetization is observed. This decrease is of PM-like type not right above $\mathrm{T}_{\mathrm{B}}$, but at larger $\mathrm{T}\left(k_{B} T>E_{B}\right)$. Because of the above reasons, some authors do not associate directly the maximum in the curves to $T_{B}$ and prefer a different definition [9]. Although we understand such discrepancy and share the necessity of finding a more precise formalism for relating the shape of the ZFC curves to the particles' characteristics, we associate in this work the maximum of the ZFC curve with $T_{B}$ because it is the usually preferred when dealing with experimental data. It is important to emphasize these aspects when dealing with the magnetic properties of SPM systems, remarking that $T_{B}$ defines only a characteristic temperature value, of enormous interest for characterizing the system but not equivalent to a phase transition. In this regard, it has been intensively discussed in the literature whether strong dipolar coupling among the particles could lead to a phasetransition character at $T_{B}$ [13]. However, this is not the present case since we are dealing with a non-interacting system.

All the properties described up to now correspond to the simplest single-particle scenario, where all the particles have been treated as equivalent to each other because of being non-interacting and with parallel-aligned easy anisotropy axes, as illustrated in Fig. (6). However, a simple and ideal case as described above is rarely found in real systems, very often characterized by a random orientation of easy anisotropy axis and that only in a few cases can be regarded as non interacting. In this context, distribution of easy axis directions results in a reduction of the effective energy barriers seen by the respective magnetic moments along that direction, as schematized in Fig. (7). In addition, interparticle dipole-dipole interactions, which are long-range and anisotropic, also modify the anisotropy energy barrier of each particle. These features give place to a much more complex scenario than the one described above. Lowinteracting conditions can be described by mean-field approximations in which the single-particle barriers are slightly modified by the interaction energy. However, strongly-interacting conditions ruled by collective effects cannot be accounted by that approach, and so the use of computational techniques becomes an indispensable tool for treating systems with so many degrees of freedom. Computational techniques allow us to deal with perfectly controlled systems and a good treatment of the interactions among particles. There are two main computational approaches for dealing with the magnetic properties of interacting nanoparticle systems, namely the Monte Carlo (MC) and Langevin Dynamics (LD) methods. Both methods are complementary for the study of a nanoparticle system: MC simulations are very adequate to treat long-time (static) magnetic properties in complex interacting systems [13] but do not have associated a physical time; LD methods, on the contrary, are very precise for modeling the dynamics of the magnetic moments [14] but are not adequate to simulate static processes. Promising approaches trying to relate the more physical LD-time to the MC time steps have been developed in recent years, see for example the works by U. Nowak et al. [15] and X.Z. Cheng et al. [16].

Finally, it is worthy to recall again the several simplifications assumed in this introduction to superparamagnetism, where we have considered very simple and ideal characteristics for the particles. In real systems there are always several dispersive -often uncontrolledcharacteristics (inhomogeneities in the particles' composition; temperature-dependent $K$ and $M_{S}$; size/anisotropy distribution; etc), characteristics all that confer additional uncontrolled degrees of freedom to the already very complex system, and so make very hard to interpret the magnetic measurements in order to characterize their properties. ${ }^{3}$ That is the reason why we have focused on a very simple scenario which, on the other hand, it is already complex enough to make absolutely necessary the use of computational techniques for its study.

\footnotetext{
${ }^{3} \mathrm{~A}$ particularly relevant difference between superparamagnetism and paramagnetism is found on the strong temperature-dependence of the particles' magnetic moments in contrast with the essentially temperatureindependent atomic magnetic moments. Thus, PM systems are usually characterized by a linear evolution of the inverse of the magnetic susceptibility $v s$ temperature, whereas SPM systems can deviate significantly from this linear behavior (see for example D. Serantes et al., Chem. Mater. 22 (2010) 4103, and references therein for further details).
} 


\section{Random easy axes}
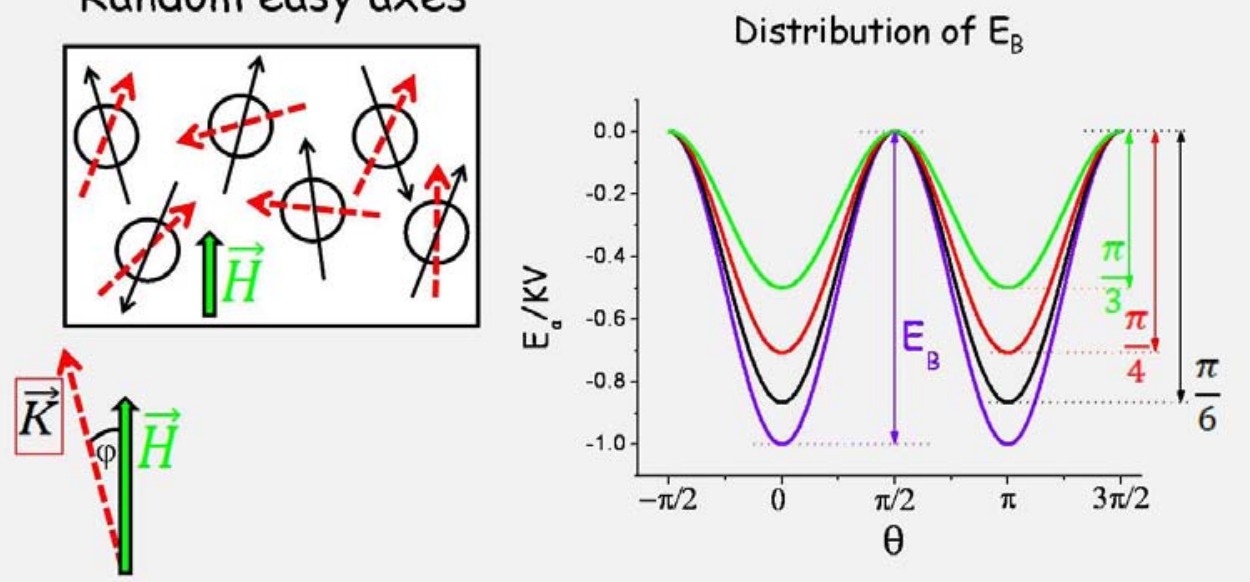

Fig. (7). Left panel: schematic drawing of a random easy axes distribution. Right panel: effective energy barriers seen by the magnetic moments along a given direction, depending on the angle between the easy axis and such direction.

\section{MONTE CARLO METHOD}

Monte Carlo (MC) methods are a type of numerical simulation techniques based on the generation of random numbers [17]. MC methods are utilized to solve complex square with the circle held inside. The graph shows the fraction between points laying in each geometrical figure as a function of the amount of random events generated, pointing out that the approximation to determine $\pi$ from the random points areal ratio improves with $\mathrm{N}$.

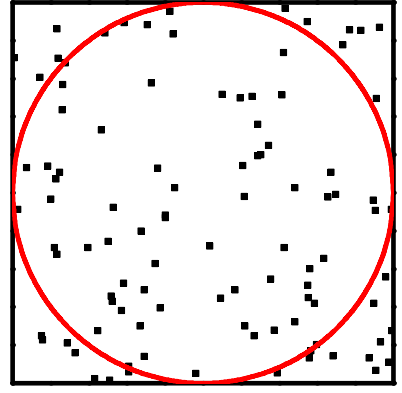

$\mathrm{N}=100$

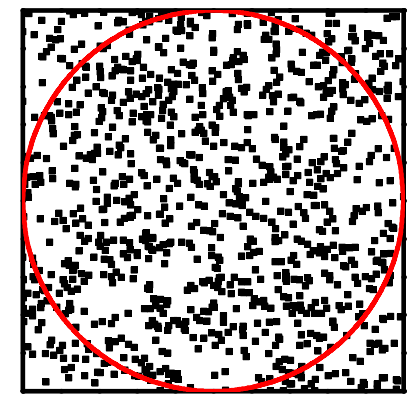

$\mathrm{N}=1000$

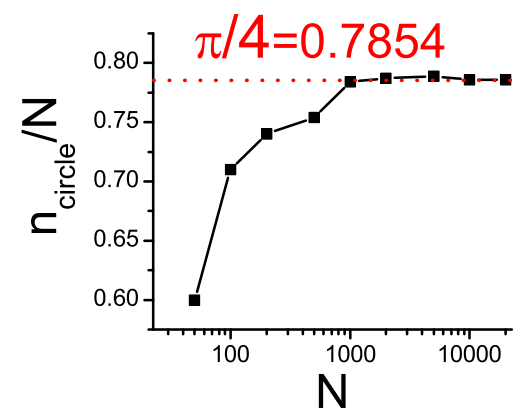

Fig. (8). Use of random events to calculate the value of $\pi$ from the area of a circle, illustrating the role of the amount of events in the precision of the result.

problems with large degrees of freedom: the features of a particular problem are represented by probabilities, and the $\mathrm{MC}$ technique generates large amounts of random numbers and counts the fraction of them obeying some conditions. The way of counting and the conditions imposed define the numerical algorithm. A simple example to illustrate the functioning of a MC method is the calculus of $\pi$ from the area of a circle. If placing a circle of radius $r$ into a square of side $2 r$ and randomly generating $N$-points into the square, the fraction of them laying inside the circle $\left(n_{\text {circle }}\right)$ will be equal to the proportion between areas, and so it is easy to obtain $\pi=4 \frac{n_{\text {circle }}}{N}$. The MC calculation of the area will be more precise the larger the amount of points (events) generated, as illustrated in Fig. (8).

The two drawings plotted in Fig. (8), stand for two examples of random generation of events $(\mathrm{N}=100$ and $\mathrm{N}=1000)$ into the

\subsection{Metropolis Algorithm}

We aim in this work to describe how to use a MC method to simulate the magnetic properties of a magnetic nanoparticle system with a large amount of degrees of freedom, as described in the previous Section 2. Specifically, we want to know how the orientation of the magnetic moments of the particles evolves as a function of different parameters (temperature, applied magnetic field, etc), i.e. how they behave as a function of the different energies involved. To simulate such processes we impose to the system some known conditions and determine its configuration from a random generation of events as evaluated under those conditions. The processes we want to simulate are essentially quasistatic, and so the conditions ruling the system can be based on minimum energy arguments on the following manner: i) the energy of the system under certain conditions is evaluated, ii) under the variation of an external parameter the internal degree of 
freedom changes, and thus the energy is reevaluated, and iii) the difference in energy is used to construct a probability function, and the change of configuration of the system is accepted or not from the random generation of events applied to such probability under a given algorithm.

It turns out that this problem is much more complex than calculating $\pi$ from the ratio between the areas of the circle and square as described above, where the randomly generated events are equally probable and so the algorithm for solving the problem is just to count 1 if the points lay inside the circle and 0 otherwise. If we apply the same procedure to simulate the orientation of the magnetic moments with temperature, i.e. if we randomly generate new possible orientations and evaluate its feasibility to occur, we will find that most of the trials are highly improbable and so rejected, and only those with energy comparable to the previous state will have some chance of being accepted. For example, for simulating the new possible orientation of a particle' magnetic moment, initially at an angle $\theta_{i}$ (Eq. (6)), the new trial configuration $\theta_{f}$ can be chosen totally at random, unrelated to $\theta_{i}$, or by considering a slight variation after the actual configuration, so that $\theta_{f}=\theta_{i}+\delta \theta$ with $\delta \theta$ small. In the first case many trials will be very unfavorable and therefore rejected; while for the latter a higher acceptance ratio is expected. It becomes therefore crucial, in order to avoid the wasting of computational time and for optimizing the simulation, to be able to select the new trial configurations among the most likely probable paths. This can be done if considering a Markov chain of events (configuration of one state depends only on the previous one), with the trial state being close in energy to the current one.

The key-points for treating the present problem are, therefore, i) the selection of the trial configurations in an efficient way, and ii) the choosing of an adequate implementation of the change from the initial state with energy $E_{i}$ to the trial next state with energy $E_{f}$. Assuming classical Boltzmann distribution, the probability of a magnetic moment to have energy $E$ at a temperature $T$ is proportional to $\exp \left(-E / k_{B} T\right)$, i.e. $\quad p(E) \propto e^{-E / k_{B} T}$. Consequently, if considering the orientation of the magnetic moments to be markovian the evolution from state $E_{i}$ to state $E_{f}$ will be proportional to the rate between final and initial states probabilities, $r_{i \rightarrow f}=p\left(E_{f}\right) / p\left(E_{i}\right)=e^{-\Delta E / k_{B} T}$, with $\Delta E=\left(E_{f}-E_{i}\right)$. This way of choosing the possible next configuration of the system as being energetically close to the actual one is named importance sampling, and is based on the detailed balance reversibility condition. This approach works very well for describing quasi-static thermodynamic processes, as intended in this work, although much care has to be taken if dealing with dynamic properties. For further details about this topic, see for example: O. Iglesias Doctoral Thesis [18], Chapter 5.

The motion of the magnetic moment of a nanoparticle from the initial state with energy $E_{i}$ to the final state with energy $E_{f}$ is often described by means of the Metropolis algorithm [19]: if $\Delta E<0$ (the new configuration is more stable energetically), the move to the new state is accepted and its energy changes to $E_{f}$, whereas if $\Delta E>0$ (new configuration is less stable than the current one), the move has still some probability $e^{-\Delta E / k_{B} T}$ to occur. To compute this probability a random number $n$ with value between 0 and 1 is generated and, if $n<r_{i \rightarrow f}$ the new conformation of the system is accepted and so it has now energy $E_{f}$, while if $n<r_{i \rightarrow f}$ it is rejected and the energy remains still $E_{i}$. The Metropolis algorithm is expressed as

$\min \left[1, e^{-\Delta E / k_{B} T}\right]$

The choice of this algorithm was motivated by its suitability to describe static properties of magnetic nanoparticle systems, as intended in this work. Other transition probability algorithms as Glauber dynamics could provide similar results for simulating $\mathrm{ZFC} / \mathrm{FC}$ magnetization curves [20]. Glauber dynamics, originally applied to the 1D-Ising model, mainly differs from Metropolis in that minimizing the energy is not always accepted as change. For more details on this and other algorithms as heat-bath see for example the work by K. Binder [17]. It is also interesting to note ref. [21], where the governing algorithm is constructed as a combination of different MC trial-step choices.

The Metropolis MC method can be used to simulate the evolution of the magnetization of a system of magnetic nanoparticles as a function of different parameters. We describe here a MC method based in this procedure (see for example refs. [21-32]): the simulated system consists on an assembly of $\mathrm{N}$-particles contained inside a unit cubic cell (side $L$ ), which is replicated by using periodic boundary conditions in order to resemble a large and homogeneous system. The simulations are always done in two parts: in the first one the spatial distribution of the particles is set, and in the second part the particles are characterized by their attributes (size, anisotropy, magnetic moment) and the evolution of the system is simulated as a function of the desired parameters. The positions set in the first part are kept fixed during the simulation of the magnetic properties. Next we give a brief description of the generalities of the MC method.

\subsection{Spatial Arrangement}

The procedure used to achieve the spatial distribution of the particles varies depending on the type of system we want to simulate: for ordered structures (chains of particles, crystalline structures, etc) the particles are directly placed into the unit cubic cell under the desired structure, whereas for setting the spatial arrangement of liquid-like systems (e.g. a frozen ferrofluid) a relaxation algorithm has to be used. 


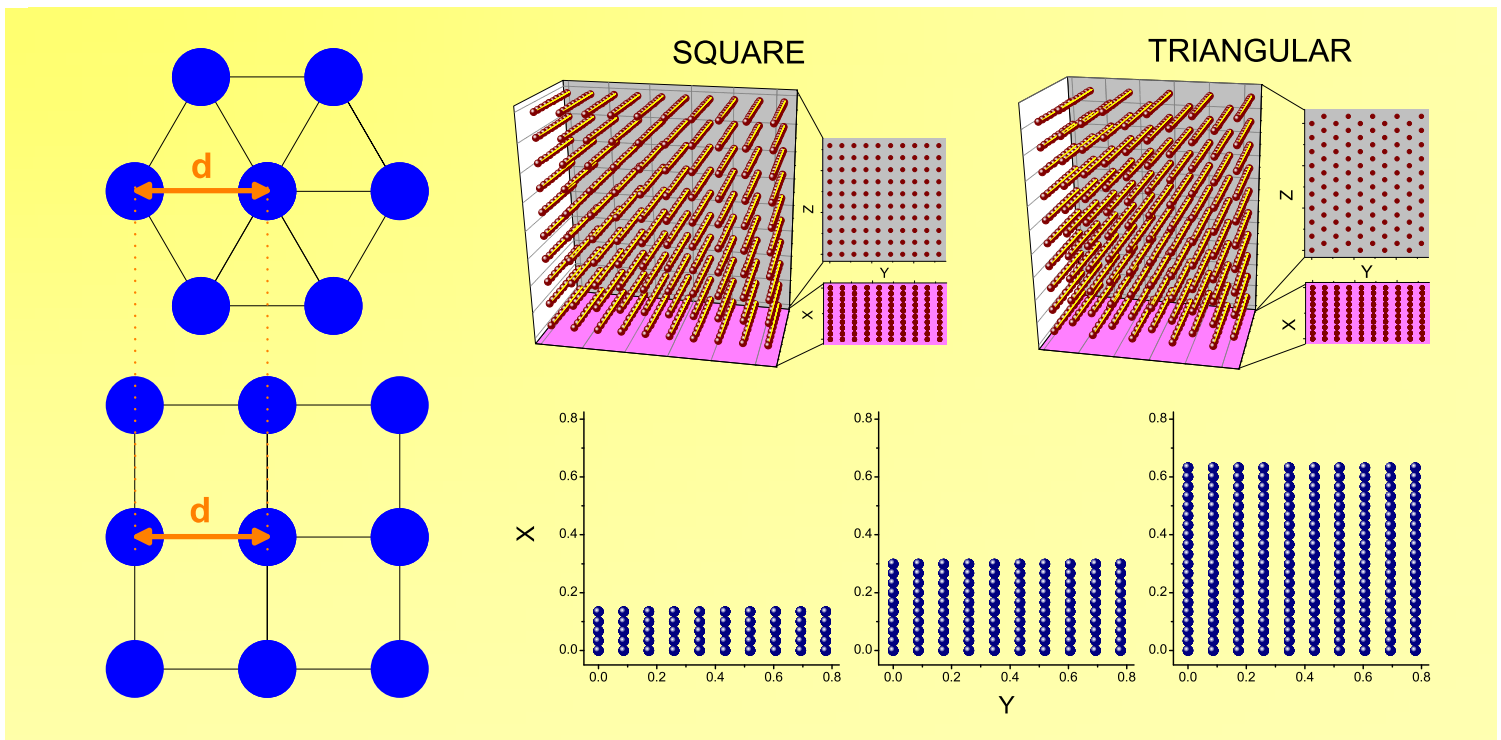

Fig. (9). Scheme of parallel-aligned chain-like structures hexagonally and squared distributed, and different lengths.

In Fig. (9) we show some chain-like structures obtained by directly placing the particles into desired regular positions. They resemble one-dimensional columnar parallel chains of magnetic nanoparticles under different spatial arrangement (square, hexagonal) and different lengths along the X-axis. The study of such type of structures is at the center of much research nowadays for the basic study of the competition between the enhanced anisotropy and magnetostatic interactions [31-35]. The magnetic properties of such chain-like systems exhibit a good analogy to the behavior of ferromagnetic nanowires [36].

For simulating disordered systems as ferrofluids with liquid-like structure [37] the positions of the particles are not directly generated and so we use a Lennard-Jones pair potential $\left(v_{L J}\right)$ to distribute the particles. During the simulation the particles can move freely in space, but their trial positions are markovian-linked to the actual one and so the liquid-like structure is more quickly obtained. An example of liquid-like structure is shown in Fig. (10), together with the corresponding correlation function $g_{2}$.

For treating the long-range dipolar interactions the Ewald summation is used as in ref. [13], using for the simulations periodic boundary conditions based on the repetition of the unit cubic cell. Once attained the desired spatial distribution, the interparticle distances and their positions are used as inputs for the next part of the simulations, in which they are kept fixed.

\subsection{Superparamagnetic (SPM) Properties}

Once the spatial distribution of the particles is achieved the next step is to characterize them with their main physical properties, namely volume, magnetic moment and magnetic anisotropy. Following the model described in Section 2, magnetic anisotropy is considered of uniaxial type, and both magnetic moment and anisotropy are assumed to be proportional to the particle volume. Therefore, the important parameters to characterize the particles are their volume and the orientation of the anisotropy easy axis.

The volume is taken into account by means of the related sample concentration of the system, $c$, preferable to determine experimentally and so better to compare with experimental results. For the sake of simplicity we assume the same monodisperse system as in the previous section, in order to have the less uncontrolled parameter-dispersity as possible. If defining $c$ as the fraction of the volume occupied by all the particles $\left(\sum_{i}^{N} V_{i}\right)$ over the total system volume $\left(V_{T}=L^{3}\right)$, then the relationship between particle size and volume sample concentration is

$c=\frac{\sum_{i}^{N} V_{i}}{L^{3}} \equiv \frac{N V}{L^{3}}$

The orientation of the anisotropy easy axes of the particles is a parameter that remains fixed along the whole simulation process, and hence its value must be carefully selected due to its strong influence on the magnetic properties of the system. For studying SPM properties we have assumed a random easy-axes distribution as schematized in Fig. (7), constraint that works quite well for describing real systems as frozen ferrofluids or solid matrices [38]. Once the easy axes are placed, the simulation of the magnetic evolution of the magnetic moments of the particles can start, whose initial orientation can also be randomly distributed. The magnetic properties of the particles are accounted by their magnetic moment $\vec{\mu}$ and magnetic anisotropy $\vec{K}$, under the temperature/magnetic field constraints applied to the system, which determine its energy. In real systems, the evolution between an initial state 

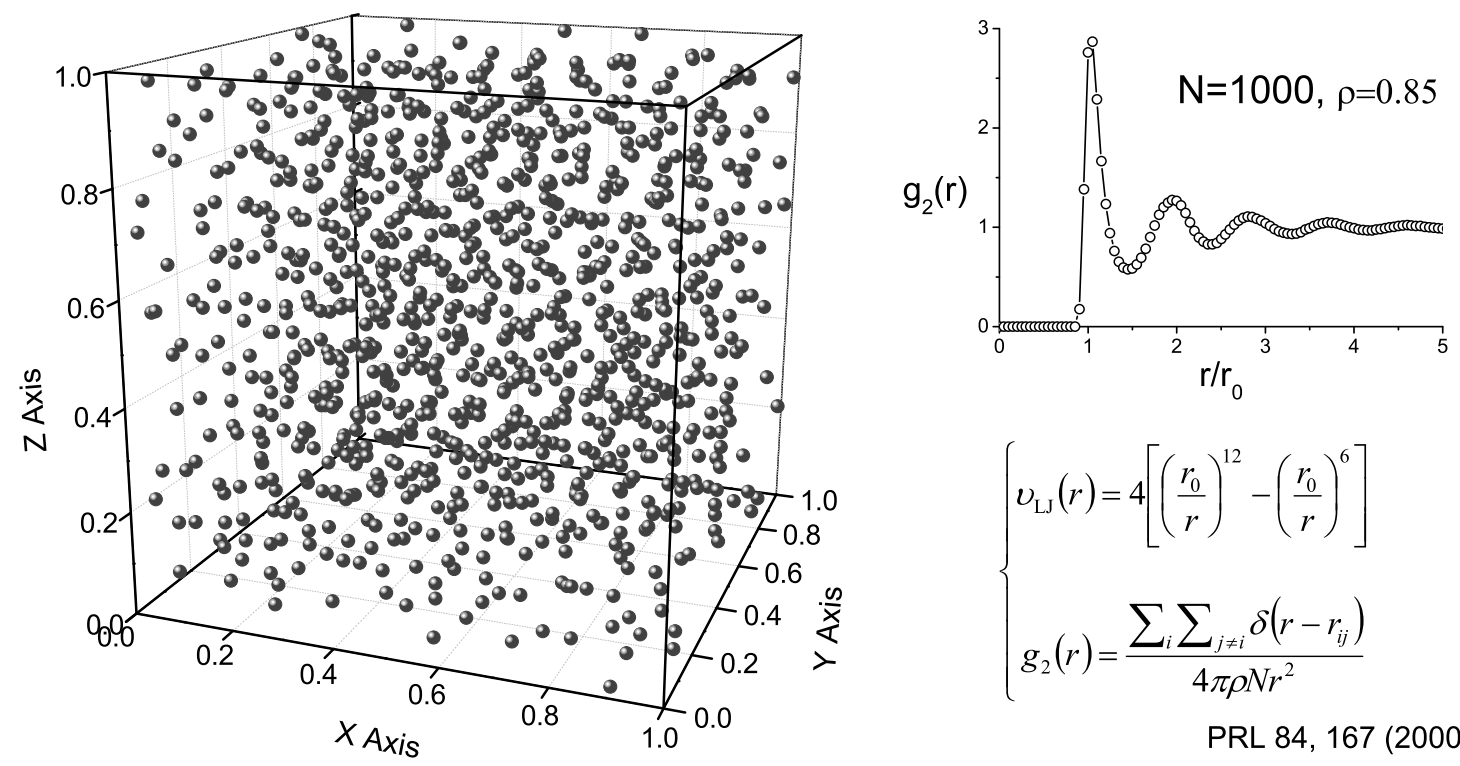

$$
\left\{\begin{array}{l}
v_{\mathrm{LJ}}(r)=4\left[\left(\frac{r_{0}}{r}\right)^{12}-\left(\frac{r_{0}}{r}\right)^{6}\right] \\
g_{2}(r)=\frac{\sum_{i} \sum_{j \neq i} \delta\left(r-r_{i j}\right)}{4 \pi \rho N r^{2}}
\end{array}\right.
$$

PRL 84, 167 (2000)

Fig. (10). Liquid-like distribution of $\mathrm{N}=1000$ particles into a unit cubic cell and correlation function $g_{2}(r)$. The correlation function and the Lennard-Jones pair potential are also indicated.

with energy $E_{i}$ and a final state with energy $E_{f}$ occurs at a certain time interval, in which the event (reorientation of the magnetic moments in this case) can be described by a probability distribution function. These time-dependent processes can be simulated by giving the system a certain amount of opportunities to occur, i.e. attempts to change the configuration. These attempts define the computational time and are called Monte Carlo steps. The MC step constitutes, therefore, the computational equivalence to real time units. In our simulations one MC step is defined as $N$ trials given to a system of $N$-particles to change its configuration.

The simulation of a physical process consists in varying a desired magnitude (temperature, magnetic field, etc) under a certain protocol and evaluating the energy in the new state after a certain number of MC steps, accepting or neglecting the new configuration under the chosen algorithm. For example, to simulate a ZFC process for a $N$-particle system, the system is first cooled down in zero field from a high temperature by following a constant temperature vs MC steps variation ratio down to a very low temperature. In every MC step, one particle is selected at random and a new orientation of its magnetic moment is generated and accepted under the Metropolis algorithm $\min \left[1, e^{-\Delta E / k_{B} T}\right]$. This scheme is repeated $N$-times in each MC step. Once the very low temperature is reached, a small magnetic field is applied and the process continues now while heating the sample ${ }^{4}$.

\footnotetext{
${ }^{4} \mathrm{~A}$ thermalization is performed previous to any thermomagnetic simulation (ZFC, FC, magnetization vs. field) in order to rapidly reach a stable configuration. The reason for doing this is to reduce the initial instability of the system right after randomly generating the orientation of the magnetic moments and easy-anisotropy axes, which would take a vary large amount of MC steps to stabilize during the simulation process. Instead, if the system is initiated at a very high temperature and cooled in large temperature jumps down to the starting process temperature the system relax rapidly and reaches a energetically stable configuration
}

The same procedure applies for simulating the magnetization vs magnetic field $\mathrm{M}(\mathrm{H})$ curve, just being different the parameter to vary after a certain amount of MC steps. A $\mathrm{M}(\mathrm{H})$ curve is simulated by initially cooling the system down to the desired temperature (in zero field for our simulations), and once it is reached, a small field is applied and increased in regular intervals of field/MC steps up to a high field $H_{\max }$. Then, the field is decreased in the same manner until $-H_{\max }$ is reached; and finally increased again until reaching once more $H_{\max }$ and having completed the cycle. To illustrate the features of the MC steps resembling real time units, we show in Fig. (11) some ZFC (left panel) and $\mathrm{M}(\mathrm{H})$ (left panel) curves for fixed temperature and magnetic field variations, but different MC steps, corresponding to a system of non-interacting particles as that shown in Fig. (10).

Fig. (11a) illustrates the shift of the ZFC curve peak to lower temperatures with larger amount of MC steps, what reproduces the physical behavior described by Eq. (4): if relating the amount of MC steps with the experimental measuring time $\tau_{m}$, the longer the time interval (amount of MC steps), the smaller $T_{B}$. The $\mathrm{M}(\mathrm{H})$ curves of Fig. (11b, c) illustrate the influence of the thermal fluctuations on the hysteresis curves. For the low-temperature case, (a), the system is in the blocked state and exhibits FM-like hysteresis losses that originate from the overcoming of the anisotropy energy barrier. For the high temperature case (b), however, thermal excitations are large enough to promote the reversible jumping over the anisotropy barrier without energy losses. In both cases, it is observed a decrease in the value of the coercive field for larger time intervals (higher amount of MC steps). This tendency is also expected based on the time-dependence of the FM-like hysteretic behavior represented by the area in the $\mathrm{M}(\mathrm{H})$ curves, as discussed in 

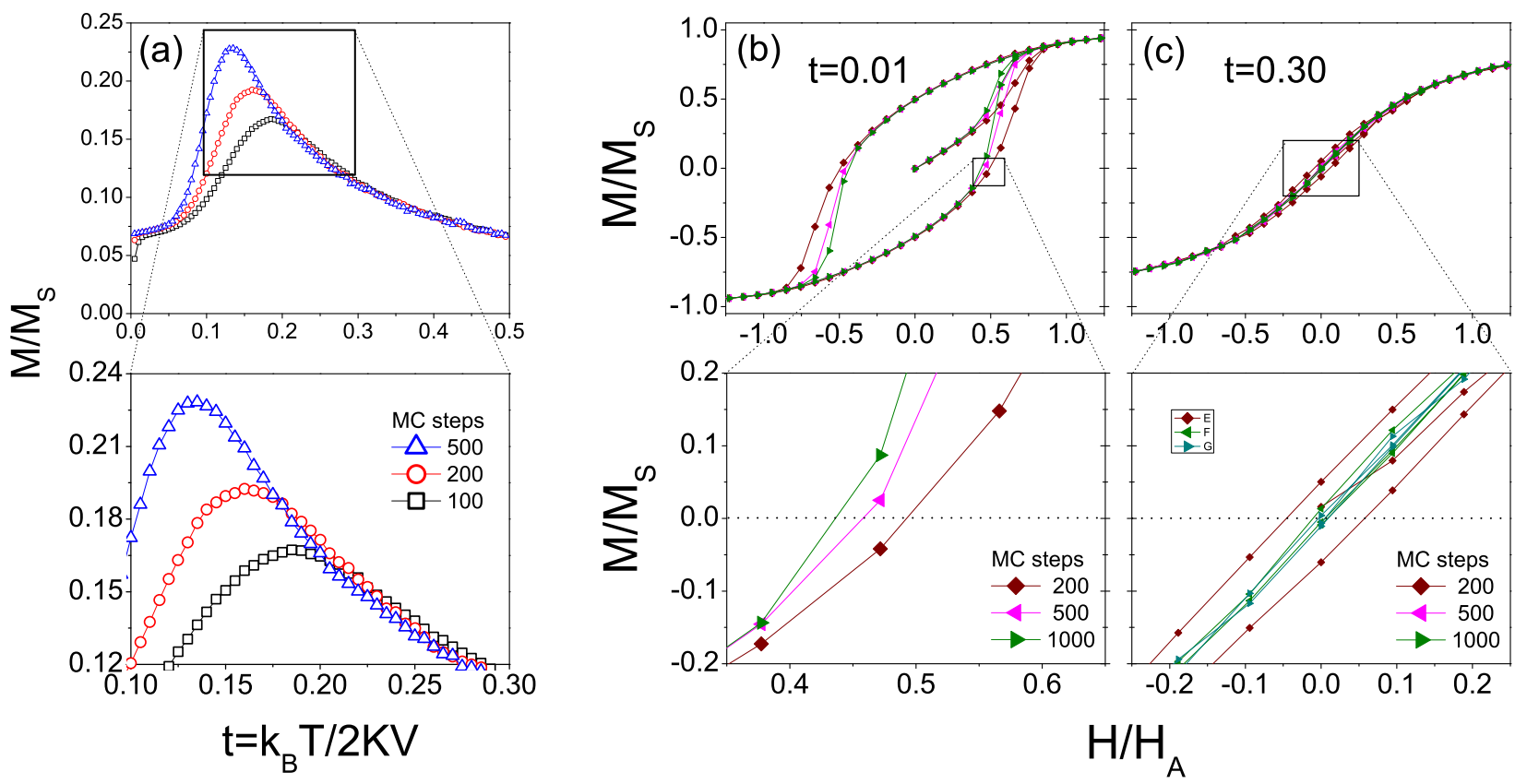

Fig. (11). (a) ZFC processes simulated for a fixed temperature interval variation, but different MC steps (100, 200, and 500). In (b, c) are plotted $\mathrm{M}(\mathrm{H})$ curves simulated at temperatures below $(t=0.01)$ and above $(t=0.30) T_{B}$, respectively. It was used a fixed magnetic field interval variation, but different MC steps $(200,500$, and 1000). In all (a ,b, c) cases, top panels show the complete processes, while bottom panels shows a magnification of the more remarkable aspects of the simulation (maximum of the ZFC curve and coercive field, respectively).

Section 2, which tends to disappear for very large times (MC steps). It is worthy to note that for the $t=0.01$ case, the anisotropy energy is already large enough in comparison with the thermal one as to observe coercivity and remanence values characteristics of the idea Stoner-Wohlfarth case $\left(H_{C} \approx 0.48 H_{A}, M_{R}=0.5 M_{S}\right)$ [12]. In fact, Stoner-Wohlfarth features can be reproduced by choosing an appropriate amount of MC steps (see ref. [28] for further details).

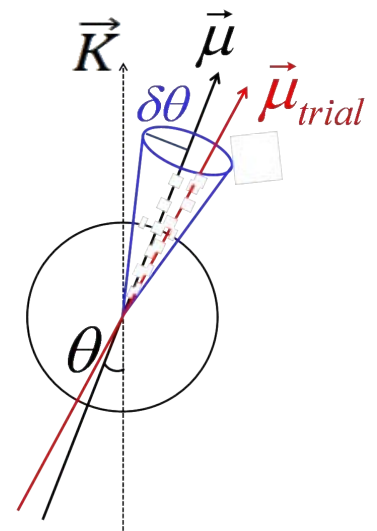

Fig. (12). Schematic drawing of the $\delta \theta$-cone used to generate the new trial orientation $\vec{\mu}_{\text {trial }}$.

Despite the physical tendency coincides with the expected for both kind of simulated $M(T)$ and $M(H)$ processes, a well-established relationship between MC steps and real time units needs still to be developed in order to establish a precise connexion between both types of measurements. Different approaches have been reported in the literature with the purpose of connecting real time with computational MC steps, as for example by using a constrained acceptance rate [22]. In latter years, an alternative approach that connects real time and $\mathrm{MC}$ steps by correlating Langevin dynamics and $\mathrm{MC}$ steps is receiving a huge attention $[15,16]$. This approach will be further analyzed in the next section.

\subsection{Trial Computational Time Steps}

It turns out from the above description of the MC technique that the choosing of the trial new configuration with energy $E_{f}$ is of utmost importance: its value determines the acceptation rate of the algorithm and so the velocity and feasibility of the simulations. In our simulations we use the solid angle restriction scheme [20]: the new trial orientation $\vec{\mu}_{\text {trial }}$ is randomly generated inside a cone of angle $\delta \theta$ around the current orientation $\vec{\mu}$. Fig. (12) illustrates this choice of the new trial orientation inside a cone of angle $\delta \theta$ around the current orientation of the magnetic moment [47]. For more information about other MC-step trial schemes and its influence on the simulation results see for example refs. [25, 26].

The $\delta \theta$-value directly rules the speed of the magnetic moments' movement, and therefore its magnitude must be carefully selected in order to ensure the adequacy of the simulations to resemble physical processes. If $\delta \theta$ is too small, the system will evolve very slowly to the quasiequilibrium configuration and we could be unable to resemble the physical process (too many MC steps would be necessary). On the contrary, if $\delta \theta$ is too large the system may relax too rapidly and make the features we want to 
study unobservable. In Fig. (13a), we show the ZFC curves of the same system obtained for different values of $\delta \theta$. It is observed a tendency similar to that displayed in Fig. (11), with the curves exhibiting a larger peak at decreasing temperature for larger $\delta \theta$-values. These results illustrate how the MC steps may perform the role of computational time, if using an appropriate choice of the trial angle.

The results plotted in Fig. (13) indicate a strong dependence of the simulated results on $\delta \theta$, hence emphasizing that much care must be taken if trying to extract time-related information about the system from the simulations: those depend not only on the amount of $M C$ steps, but also on the size of the trial angle chosen.

Fig. (13) displays also some simulations in which the $\delta \theta$-value is considered to be temperature-dependent, in the same way as described in refs. $[15,16]$. The reason for including such dependence is not related to any effort concerned to use realistic times from the MC steps or to reach more accurate time-dependence results (Figs. 11, 13a) illustrate that it constitutes a very complex task). Instead, our motivation was simply to provide the simulations with a more realistic character: it seems to us more physical to make the thermal fluctuations to be larger at larger temperatures. Our argument is based on the fact that the analogy between the time-dependence of real experiments and simulations is introduced by means of attempts to change the configuration, which are generated randomly into the cone of angle $\delta \theta$. Under this assumption, the movement of the magnetic moments is resembled by giving the system a certain amount of $\mathrm{MC}$ steps to change its configuration.

In a real system, the magnetic moments fluctuate because of the thermal energy and consequently fluctuations are reduced the smaller the temperature. In order to reproduce this characteristic in the simulations, it appears very reasonable to us to include a temperature-dependence in the value of $\delta \theta$, since it is the tool used to resemble the thermal fluctuations found in real systems. It is worth not to mention here that although there is no intention of analyzing timedependence in our simulations, however the temperaturedependence expression used has been intensively analyzed and discussed in such a context by Nowak et al. [15] and

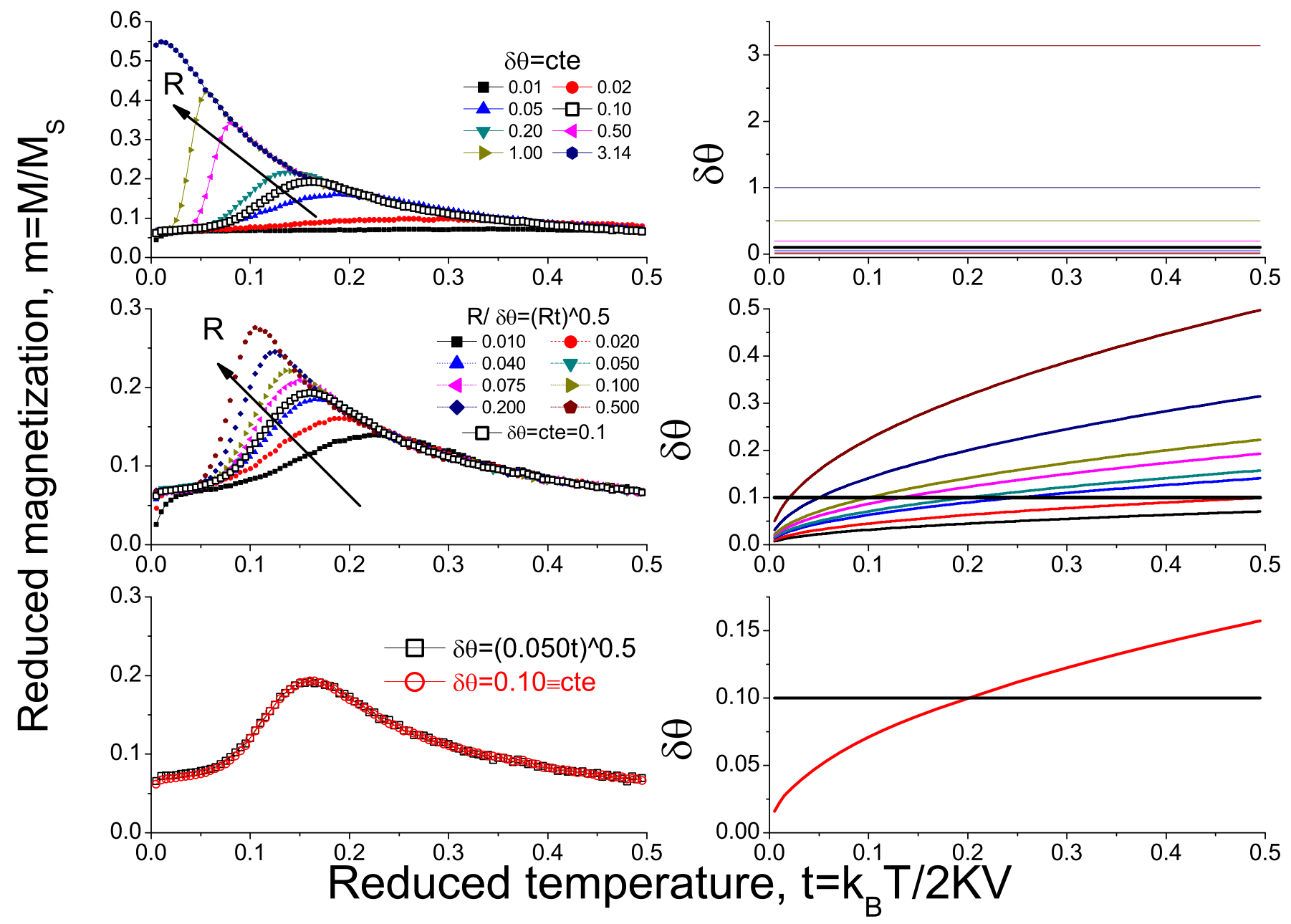

Fig. (13). Left panels: ZFC processes as a function of $\delta \theta$ where (a) corresponds to different $\delta \theta$-values constant with temperature, (b) shows different temperature-dependence for $\delta \theta$, and in (c) we compare the $\delta \theta=0.1=$ cte case, with the temperature-dependent case $\delta \theta=(0.05 t)^{1 / 2}$. In all cases, panels on the right show the temperature evolution of $\delta \theta$; the $\delta \theta=0.1=$ cte case is shown for the comparison. 
Cheng et al. [16]. The temperature dependence of $\delta \theta$ can be, re-written as $\delta \theta=(C t)^{1 / 2}$, following refs. $[15,16]$ and in usual reduced reduced temperature units of $t=k_{B} T / 2 K V$, where $\mathrm{C}$ is a constant value proportional to the particle inner characteristics (size, magnetic moment and anisotropy) and to the dynamics of the system (gyromagnetic ratio, damping parameter, measuring time). The results plotted in Fig. (13b) show that for values of $C$ of the order of 0.050 , with the results are very similar to those obtained for the $\delta \theta=0.10=$ const case, as shown in Fig. (13).

\section{SUMMARY}

We have introduced the basic features of superparamagnetism as the paramagnetic-like behaviour observed in single-domain magnetic systems above the characteristic blocking temperature, $T_{B}$. Superparamagnetic phenomenon arises from the interplay between the large magnetic supermoment resultant from the coherent rotation of the atomic magnetic moments in single-domain entities and the magnetic anisotropy energy, which defines a preferential orientation direction of significative influence on the magnetic behavior of the particles when it is comparable to the thermal energy. The direction of this anisotropy in relation to the applied magnetic field defines the response of the magnetic supermoment of the particle with temperature. In real systems, the anisotropy easy axes of the particles are usually randomly oriented, and very often interparticle dipolar interactions are not negligible, what results in a very complex scenario. In this context, we introduced the Monte Carlo technique based on the Metropolis algorithm as a powerful tool for the study of SPM properties. First, we gave a general approach to MC methods, and then described the suitability of the Metropolis algorithm for the study of SPM systems. We paid special attention to the role played by the trial angle of the MC step in the simulations.

\section{ACKNOWLEDGEMENTS}

We thank the Xunta de Galicia for financial support (Project INCITE 08PXIB236052PR), and the Centro de Supercomputación de Galicia for the computing facilities.

\section{CONFLICT OF INTEREST}

The authors confirm that this article content has no conflicts of interest.

\section{REFERENCES}

[1] Herzer G, Vazquez M, Knobel M, et al. Round table discussion: Present and future applications of nanocrystalline magnetic materials. J Magn Magn Mater 2005; 294(2): 252-66.

[2] Guimarâes AP. Principles of Nanomagnetism. Berlin: Springer 2009.

[3] Skomski R. Nanomagnetics. J Phys Condens Matter 2003; 15: R841.

[4] Dormann JL, Fiorani D, Tronc E. Magnetic Relaxation in FineParticle Systems. In: Prigogine I, Rice SA, Eds. Advances in Chemical Physics. Hoboken, NJ: John Wiley \& Sons, Inc 1997; vol. 98: pp. 283-494.

[5] Skumryev V, Stoyanov S, Zhang Y, Hadjipanayis G, Givord D, Nogués J. Beating the superparamagnetic limit with exchange bias. Nature 2003; 423: 850-3.
[6] Tartaj P, Puerto-Morales M, Veintemillas-Verdaguer S, GonzálezCarreño T, Serna CJ. The preparation of magnetic nanoparticles for applications in biomedicine. J Phys D Appl Phys 2003; 36: R182.

[7] Kechrakos D. Magnetic nanoparticle assemblies. In: Sattler K, Ed. Handbook of Nanophysics. Nanoparticles and quantum dots. UK: Taylor \& Francis 2010; vol. 3. arXiv: 0907.4417v2.

[8] Petracic O. Superparamagnetic nanoparticle ensembles. Superlattices Microstruct 2010; 47(5): 569-78.

[9] Knobel M, Nunes WC, Socolovsky LM, De Biasi E, Vargas JM, Denardin JC. Superparamagnetism and other magnetic features in granular materials: a review on ideal and real systems. J Nanosci Nanotechnol 2008; 8: 2836-57.

[10] Néel L. Théorie du traînage magnétique des ferromagnétiques en grains fins avec applications aux terres cuites. Ann Geophys 1949; 5: 99-136.

[11] Jönsson PE. Superparamagnetism and spin glass dynamics of interacting magnetic nanoparticle systems. In: Rice SA, Ed. Advances in chemical physics. Hoboken, NJ: John Wiley \& Sons, Inc 2004; vol. 128: pp. 191-248.

[12] Stoner EC, Wohlfarth EP. A Mechanism of magnetic hysteresis in heterogeneous alloys. Proc R Soc Lond A 1948; 240: 599-642.

[13] García-Otero J, Porto M, Rivas J, Bunde A. Influence of dipolar interaction on magnetic properties of ultrafine ferromagnetic particles. Phys Rev Lett 2000; 84: 167-70.

[14] Usadel KD. Temperature-dependent dynamical behavior of nanoparticles as probed by ferromagnetic resonance using LandauLifshitz-Gilbert dynamics in a classical spin model. Phys Rev B 2006; 73: 212405.

[15] Nowak U, Chantrell RW, Kennedy EC. Monte Carlo simulation with time step quantification in terms of langevin dynamics. Phys Rev Lett 2000; 84: 163-6.

[16] Cheng XZ, Jalil MBA, Lee HK, Okabe Y. Mapping the Monte Carlo scheme to langevin dynamics: a Fokker-Planck approach. Phys Rev Lett 2006; 96(6): 067208.

[17] Binder K, Heermann DW. Monte Carlo simulations in statistical physics: an introduction. Heidelberg: Springer 2002.

[18] Iglesias O. Time dependent processes in magnetic systems. PhD dissertation. Barcelona: Universitat de Barcelona 2002.

[19] Metropolis N. The Beginning of the Monte Carlo Method. Los Alamos Science Special Issue 1987; pp. 125-30.

[20] Pereira NJP, Bahiana M, Bastos CSM. Magnetization curves as probes of Monte Carlo simulation of nonequilibrium states. Phys Rev E 2004; 69: 056703 .

[21] Hinzke D, Nowak U. Monte Carlo simulation of magnetization switching in a Heisenberg model for small ferromagnetic particles. Comput Phys Commun 1999; 121: 334-7.

[22] Dimitrov DA, Wysin GM. Magnetic properties of superparamagnetic particles by a Monte Carlo method. Phys Rev B 1996; 54: 9237-41.

[23] García-Otero J, Porto M, Rivas J, Bunde A. Monte Carlo simulation of hysteresis loops of single-domain particles with cubic anisotropy and their temperature dependence. J Magn Magn Mater 1999; 203(1-3): 268-70.

[24] García-Otero J, Porto M, Rivas J, Bunde A. Influence of the cubic anisotropy constants on the hysteresis loops of single-domain particles: a Monte Carlo study. J Appl Phys 1999; 85: 2287-92.

[25] Ulrich M, García-Otero J, Rivas J, Bunde A. Slow relaxation in ferromagnetic nanoparticles: Indication of spin-glass behavior. Phys Rev B 2003; 67: 024416.

[26] Serantes D, Baldomir D, Pereiro M, et al. Magnetic fielddependence study of the magnetocaloric properties of a superparamagnetic nanoparticle system: a Monte Carlo simulation. Phys Status Solidi A 2008; 205(6): 1349-53.

[27] Serantes D, Baldomir D, Pereiro M, et al. Interplay between the magnetic field and the dipolar interaction on a magnetic nanoparticle system: A Monte Carlo study. J Non Cryst Solids 2008; 354: 5224-6.

[28] Serantes D, Baldomir D, Martinez-Boubeta C, et al. Influence of dipolar interactions on hyperthermia properties of ferromagnetic particles. J Appl Phys 2010; 108: 073918.

[29] Serantes D, Baldomir D, Pereiro M, et al. Magnetocaloric effect in magnetic nanoparticle systems: how to choose the best magnetic material? J Nanosci Nanotechnol 2010; 10: 2512-7.

[30] Serantes D, Baldomir D, Pereiro M, Hoppe CE, Rivadulla F, Rivas J. Nonmonotonic evolution of the blocking temperature in 
dispersions of superparamagnetic nanoparticles. Phys Rev B 2010; 82: 134433 .

[31] Serantes D, Baldomir D, Pereiro M, et al. Magnetic ordering in arrays of one-dimensional nanoparticle chains. J Phys D Appl Phys 2009; 42: 215003.

[32] Serantes D, Baldomir D, Pereiro M, et al. Magnetocaloric effect in dipolar chains of magnetic nanoparticles with collinear anisotropy axes. Phys Rev B 2009; 80: 134421.

[33] Lavin R, Denardin JC, Espejo AP, Cortés A, Gómez H. Magnetic properties of arrays of nanowires: Anisotropy, interactions, and reversal modes. J Appl Phys 2010; 107: 09B504-6.

[34] Lim J-H, Chae W-S, Lee H-O, et al. Fabrication and magnetic properties of $\mathrm{Fe}$ nanostructures in anodic alumina membrane. $\mathrm{J}$ Appl Phys 2010; 107: 09A334.
[35] Franco V, Pirota KR, Prida VM, et al. Tailoring of magnetocaloric response in nanostructured materials: Role of anisotropy. Phys Rev B 2008; 77: 104434 .

[36] Prida VM, Vega V, Serantes D, et al. Influence of magnetic anisotropy and dipolar interactions on magnetocaloric effect in nanostructured materials. Phys Status Solidi A 2009; 206: 2234-9.

[37] Luo W, Nagel SR, Rosenbaum TF, Rosensweig RE. Dipole interactions with random anisotropy in a frozen ferrofluid. Phys Rev Lett 1991; 67: 2721-4.

[38] Hoppe CE, Rivadulla F, López-Quintela MA, et al. Effect of submicrometer clustering on the magnetic properties of freestanding superparamagnetic nanocomposites. J Phys Chem C 2008; 112(34): 13099-104.

Received: March 30, 2011

(C) Serantes and Baldomir; Licensee Bentham Open.

This is an open access article licensed under the terms of the Creative Commons Attribution Non-Commercial License (http: //creativecommons.org/licenses/by$\mathrm{nc} / 3.0 /$ ) which permits unrestricted, non-commercial use, distribution and reproduction in any medium, provided the work is properly cited. 